\author{
Anthony Masure \\ Enseignant-chercheur en design - www.anthonymasure.com
}

\title{
Subjectivités computationnelles et consciences appareillées
}

Date

Avril 2016

\author{
Citer ce texte \\ Anthony Masure, «Subjectivités \\ computationnelles et consciences \\ appareillées $\gg$, Multitud, $\mathrm{n}^{\circ} 62$, \\ avril 2016, p. 87-96, [En ligne], \\ http://www. anthonymasure.com \\ /articles/2016-04-subjectivites- \\ computationnelles
}

Mots clés

Appareil, Assujettissement, Calcul, Computation, Conscience, Dispositif, Film, Machine, Objet, Raison, Rationnalité, Subjectivité, Sujet

\section{Auteurs}

Andreessen Marc, Agamben Giorgio, Anders Günther, Arendt Hannah, Bales Robert F., Berry David M., Cardon Dominique, Casilli Antonio A., Citton Yves, Daston Lorraine, Deleuze Gilles, Derrida Jacques, Edwards Paul N., Erickson Paul, Foucault Michel, Guattari Félix, Gödel Kurt, Gordin Michael D., Huyghe Pierre-Damien, Illich Ivan, Klein Judy_L., Lazzarato Maurizio, Leibniz Gottfried Wilhelm, Leray. Alexandre, Lemov Rebecca, LeroiGourhan André, Morozov Evgeny, Sadin Éric, Salanskis Jean-Michel, Simon Herbert A., Simondon Gilbert, Sturm Thomas, Varenne Frank, Vilayphiou Stéphanie, von Neumann John 


\section{Anthony Masure \\ Enseignant-chercheur en design - www.anthonymasure.com}

\section{Subjectivités computationnelles et consciences appareillées}

\section{Résumé}

Cet article revient sur la notion de « subjectivité computationnelle » formulée par David $M$. Berry visant à développer une approche critique des technologies numériques. Afin de comprendre les implications philosophiques d'un tel rapprochement entre « subjectivation» et «computation», nous reviendrons tout d'abord, via Leibniz et Hannah Arendt, sur l'émergence des sciences modernes qui visent à faire du « sujet » classique une entité calculante. Nous verrons ensuite comment les sciences «comportementales » ont influencé la conception des ordinateurs en substituant à la raison humaine des modélisations rationnelles déléguées à des machines. Pour sortir de l'impasse d'une déshumanisation annoncée dès la fin des années 1970 par des auteurs comme Ivan Illich ou Gilles Deleuze, nous envisagerons enfin la «subjectivation» comme un processus qui ne nécessite pas qu'il y ait sujet. Le concept d'« appareil », tel que le propose Pierre-Damien Huyghe à propos de la photographie et du cinéma, peut ainsi être étendu aux machines computationnelles pour penser de possibles «consciences appareillées».

Que faire avec nos machines à traiter de l'information? La question pourrait sembler triviale si chaque journée ne nous fournissait pas son lot de complaintes dirigées contre des objets technologiques sans lesquels on vivrait mieux (les «humanités» sans le numérique, les taxis sans les plateformes, les rencontres sans les écrans, la photographie sans les smartphones, etc.). Il est vrai, par exemple, que le digital labor ${ }^{1}$ (travail dissimulé), «l'économie de l'attention ${ }^{2} »$ (prolongeant le capitalisme des biens de consommation) ou encore le développement des «applications» (remplaçant les logiciels ${ }^{3}$ ) semblent faire de nos vies des entités inertes vouées au divertissement ${ }^{4}$ ou au service ${ }^{5}$. Dans cette logique, nos subjectivités seraient devenues «computationnelles » en tant qu'elles adhéreraient sans réserve à tous les dispositifs d'assujettissement et d'asservissement des pro-grammes ( «écrits à l'avance ${ }^{6}$ »). Il faut pourtant nous y résoudre: nous n'habiterons pas un autre monde. C'est pourquoi ces inquiétudes légitimes ne doivent pas nous réduire à l'impuissance face à des objets qui se conduiraient de façon autoritaire, sans nous. Faire «avec» les techniques computationnelles signifie que nous n'en avons pas fini avec les «boîtes noires», mais que d'autres directions sont possibles que 
celles qui sont dominantes économiquement. Autrement dit, ce qu'une technique nouvelle emporte de singulier est toujours à revendiquer, à conquérir, à cultiver.

Afin de rendre effective une telle visée, David M. Berry (philosophe et théoricien des nouveaux media) propose l'expression de «subjectivités computationnelles ${ }^{7}$ » en mettant l'accent sur la maîtrise pratique et collective des langages des programmations. Avec sa notion d'«itéracie numérique », Berry ouvre des perspectives stimulantes sur une reconfiguration de la notion de «sujet » par les possibilités du numérique:

S'appuyer sur la technologie d'une façon plus radicalement décentrée, dépendre d'appareillages techniques pour remplir les blancs dans nos esprits et pour établir de nouvelles connexions entre les connaissances - cela conduirait à modifier notre compréhension de la connaissance, de la sagesse et de l'intelligence elles-mêmes. Cela entraînerait en effet un décentrement radical, dans la mesure où le sujet [...], rempli de culture et d'une certaine conception de la rationalité, cesserait simplement d'exister. [...] Cette nouvelle subjectivité se caractérise par le fait d'être computationnellement communicative, sachant obtenir, traiter et visualiser les informations et les résultats de façon rapide et efficace ${ }^{8}$.

Cet article se propose de revenir sur cette expression complexe de «subjectivités computationnelles» afin d'étudier ses implications politiques et philosophiques. Ce type de démarche ne va pas sans poser de nombreux problèmes. Si le «sujet » n'existe qu'à l'intérieur de mondes techniques sans extériorité, quelles «subjectivités computationnelles» pouvonsnous revendiquer? En quoi le concept d'«appareil», tel que le pense Pierre-Damien Huyghe, permettrait-il de dépasser l'opposition classique entre le « sujet » et l'« objet»?

\section{L'isolement du sujet dans les sciences modernes}

Dans la Monadologie, Leibniz donne une vision de ce que pourraient être des «subjectivités computationnelles » idéales, à savoir des entités autonomes parfaitement «réglées » par un système extérieur. Comme le dit Leibniz, « les Monades n'ont point de fenêtres, par lesquelles quelque chose y puisse entrer ou sortir ${ }^{9}$ ». Elles ne peuvent dépendre l'une de l'autre que par l'intermédiaire d'un «Architecte» (Dieu) «réglant dès le commencement des choses ${ }^{10}$ » leur agencement. Ce «meilleur des mondes possible ${ }^{11}$ » où la raison est réduite à un calcul peut ainsi faire écho à notre époque contemporaine, où le credo de nombreuses startups consiste à «faire du monde un meilleur endroit ${ }^{12}{ }$. En faisant de nos existences des entités dont chaque aspect peut être découpé, isolé et pris en charge par cet «assujettissement computationnel », ces «plateformes» ayant pour finalité de «régler » le désordre humain risquent de nous enfermer dans une "société de l'anticipation ${ }^{13}$ ", où nos désirs et décisions «humains, trop humains » seront entièrement délégués à des machines, en un nouvel «optimisme » nourri d'optimisations et prometteur d'une nouvelle harmonie computée par des appareils supérieurs à toute intelligence individuelle. 
Ce scénario de la souveraineté isolée du «sujet » classique aura été constitutif d'une certaine forme de modernité, celle des avancées scientifiques liant la connaissance du monde extérieur au développement des instruments d'observation (télescope, etc.) plus performants que les cinq sens. Pour Hannah Arendt, contrairement à la géométrie platonicienne liant la faculté de connaître à la perception, la géométrie soumise au «traitement analytique [est celle] où se reconnaît l'idéal moderne qui est de réduire [l'entendement] en symboles mathématiques ${ }^{14}$ ". Dans cet espace entièrement construit par les lois logiques des «mathématiques pures», nous dit Arendt, l'être humain ne peut avancer que dans ce qu'il a déjà démontré, «sûr de n'y rencontrer que [lui]-même ou de n'y rien voir qui ne puisse se amener à des schémas qu'il portait déjà en lui ${ }^{15}{ }^{1}$ :

Dans ces conditions d'éloignement, tout assemblage d'objets se transforme en simple multitude, et toute multitude, si désordonnée qu'elle soit, si incohérente, confuse qu'elle soit, peut se ranger en schémas et en figures tout aussi valables, tout aussi dépourvus de sens, que la courbe mathématique que l'on peut toujours trouver, comme Leibniz le remarquait, entre des points jetés au hasard sur une feuille de papier ${ }^{\mathbf{1 6}}$.

C'est pourquoi, selon Arendt, à l'époque moderne, «les êtres humains ne sont plus [...] que des animaux [animale rationale] capables de «calculer les conséquences ${ }^{17}$ ». Or, cette réduction des sciences en principes mathématiques [la reductio scientiae ad mathematicam] semble avoir trouvé son parfait prolongement dans les machines numériques, qui calculent mieux que ne le feraient un être humain ou une machine analogique ${ }^{18}$. C'est pourquoi il faut resituer les problématiques liées aux «subjectivités computationnelles» dans le temps long de l'extériorisation technique des fonctions biologiques ${ }^{\mathbf{1 9}}$. Il n'est donc pas possible de penser une subjectivité humaine qui préexisterait aux machines.

\section{Les fictions de la modélisation des rationalités}

Dans le contexte de la Guerre Froide et de la crainte d'une escalade nucléaire pouvant mener le monde à sa perte ${ }^{20}$, le développement effectif de l'externalisation du calcul dans les machines «rationnelles» s'opère par une volonté de mettre à plat les incertitudes de la «raison » humaine (faillible, imprévisible, soumise aux sentiments, etc.). Comme le démontre le passionnant ouvrage de synthèse Quand la raison faillit perdre l'esprit, les recherches qui mobilisèrent «les plus grands esprits de l'époque ${ }^{21} »$ trouvèrent rapidement des applications dans le champ économique:

[Afin d'améliorer les modélisations de la prise de décision de l'Homo Economicus], la tâche consist[ait] à remplacer la rationalité globale de l'homme économique par un comportement rationnel compatible avec l'accès aux informations et aux capacités de calcul ${ }^{\mathbf{2 2}}$.

La réduction des contingences humaines à des protocoles séquentiels aura permis d'«associer la rationalisation économique à la rationalité algorithmique ${ }^{23}$ ». L'étude de cette période 
historique nous montre qu'il existe ainsi une multitude de liens entre la mise à plat des processus de décision propres aux êtres humains et la structure logique des machines numériques. Or, une telle volonté repose sur une fiction d'autonomie et d'isolement comparable aux Monades de Leibniz ou à l'idéal moderne décrit par Arendt des mathématiques analytiques. En effet, dans un étrange jeu d'auto-détermination, les protocoles d'observation scientifiques des «sujets » étudiés pendant la Guerre froide étaient directement issus des contraintes structurelles des «situations» d'observation et de la mise en forme des données (puissance de calcul limitée, observateur solitaire ${ }^{24}$, etc.). Cet étrange «monde clos $^{25}$ » rejouant en miniature la psychose de la Guerre froide et ayant pour but de dégager des «modèles » pouvant être appliqués à des populations entières montra rapidement ses limites: les arbres de décision et les méthodes logiques ne pouvaient être dupliquées à des échelles plus larges et à des sociétés non occidentales.

Il n'empêche: la volonté demeura vive de remplacer le «bol de mélasse ${ }^{\mathbf{2 6}}$ » de la pensée humaine par la rationalité abstraite des «matrices» puis des programmes informatiques (logiques, présumés infaillibles, non soumis à des contingences, etc.). Ces sujets «fictifs» auront pu être inclus dans les machines à calculer du fait même de leur « réduction» mathématique. Nous pouvons ainsi dire que le «sujet» computationnel est directement issu des conditions matérielles dans lesquelles les sciences comportementales ont été traduites en algorithmes puis en programmes. Il nous faut alors reprendre ce que disait David M. Berry à propos d'un «décentrement radical, dans la mesure où le sujet [...], rempli de culture et d'une certaine conception de la rationalité, cesserait simplement d'exister ${ }^{27}$ ». Ce n'est pas seulement la rationalité du sujet qui disparaît, mais également sa «raison », sa forme propre de pensée, remplacée par une «rationalité » mathématique calculant pour lui les décisions à prendre.

\section{De «l'assujettissement social » à «l'assujettissement machinique "}

Dès la fin des années 1970 , des auteurs comme Ivan Illich avec sa notion de « convivialité ${ }^{28}$ », Gilles Deleuze et ses réflexions autour des sociétés de contrôle ${ }^{29}$ ou encore Félix Guattari avec ses écrits autour de la subjectivité et du machinique ont développé des réflexions décisives autour des risques liés au développement de nos subjectivités face à la déferlante aveuglante et aliénante des technologies numériques:

La subjectivité demeure aujourd'hui massivement contrôlée par des dispositifs de pouvoir et de savoir qui mettent les innovations techniques, scientifiques et artistiques, au service des figures les plus rétrogrades de la socialité. Et pourtant, d'autres modalités de production subjective celles-là processuelles et singularisantes - sont concevables. Ces formes alternatives de réappropriation existentielle et d'auto-valorisation peuvent devenir demain la raison de vie des collectivités humaines et des individus qui refusent de s'abandonner à l'entropie mortifère caractéristique de la période que nous traversons ${ }^{30}$. 
Sans avoir le temps ici de revenir en détail sur l'actualité de ces critiques, il nous faut tout de même distinguer entre deux régimes de gouvernement des subjectivités. Le premier est celui que Maurizio Lazzarato, à la suite de Gilles Deleuze et Félix Guattari, appelle «assujettissement social»:

L'assujettissement social est un processus de subjectivation, au double sens où les membres d'une société sont «assujettis» à un ordre social dont ils assurent la reproduction [...] et où ils forment des «sujets » individués de façon relativement stable et intégrée, des «personnes» morales non-schizophréniques, se comportant d'une façon relativement stable et responsable ${ }^{\mathbf{3 1}}$.

À ce premier régime recoupant de multiples formes de pouvoir et de domination s'ajoute désormais ce que Deleuze et Guattari ont appelé l'«assujettissement machinique » pour désigner des types de procédures où le conditionnement ne passe plus par du langage (du symbolique) mais où il s'agit, par le biais de procédures programmées, de faire fonctionner «les composantes de la subjectivité [...], non encore assignable[s], à un sujet [...], comme [...] des éléments d'une machine ${ }^{32}$ ". Il est ainsi, par exemple, d'un guichet automatique demandant de répondre servilement à ses injonctions pour pouvoir retirer de la monnaie:

\begin{abstract}
Ici, ce n’est plus le sujet qui agit, mais le «dividuel» qui fonctionne comme un rouage, un élément «humain» qui s'agence avec les éléments «non humains» de la machine sociotechnique constituée par le réseau bancaire. L'assujettissement social mobilise les individus, tandis que l'assujettissement machinique active les «dividuels» en tant qu'opérateurs, agents, éléments ou pièces humaines de la machine sociotechnique ${ }^{33}$.
\end{abstract}

Ce découpage de l'in-dividu en entités («dividuels») qu'aucune subjectivité ne viendra articuler pour les relier au sein d'une conscience douée de raison (à la manière des sujets kantiens) permet de réduire, par des opérations de «discrétisation ${ }^{34}$ » (pointage), tous les aspects d'une existence à quelques caractéristiques comparables entre elles. Ainsi, si l'on n'y prend pas garde, les «subjectivités computationnelles», du fait de ces divisions, sont les proies idéales d'un «asservissement machinique» faisant d'elles des entités dépourvues d'existence authentique. De nombreux «dispositifs ${ }^{35}$ » relevant du «capitalisme cognitif» ont ainsi pour but de tracer, quantifier et valoriser chacun de ces «dividus» (inputs) afin d'adresser à des sujets dépourvus de raison des réponses «sur mesure » $\left(\right.$ outputs $^{36}$ ) et autres promesses de «contenus pertinents». Comme le dit Evgeny Morozov: «Quelqu'un, quelque part, finira par vous évaluer en tant que passager, hôte de maison d'hôtes, étudiant, patient, client ${ }^{37}$ ». Comment échapper à de tels procédés sans perdre de vue les fantastiques possibilités induites par le numérique? Autrement dit, alors que le «sujet » kantien, doué de raison et prenant part à la vie politique, trouvait des formes d'individuation par un accès à la connaissance via l'alphabétisation et les media imprimés, comment faire pour que son «décentrement» (David M. Berry) au sein des systèmes numériques ne débouche pas sur des formes d'aliénation incontrôlables?

\title{
Des subjectivités sans sujets
}


Un premier élément de réponse consiste à revenir au texte de David $M$. Berry qui part d'une fin des «sujets» doués de raison individuelle pour construire une réflexion autour des «subjectivités » computationnelles. Ce qui nous importe dans ce déplacement est la possibilité - paradoxale et difficile - de penser une subjectivité sans sujet. Une telle hypothèse est développée par Gilles Deleuze dans un entretien à propos de Michel Foucault:

\begin{abstract}
La subjectivation, c'est la production des modes d'existence [...]. Foucault dit: «un art de soimême qui serait tout le contraire de soi-même... » S'il y a sujet, c'est un sujet sans identité. La subjectivation comme processus, c'est une individuation, personnelle ou collective, à un ou plusieurs. Or il y a beaucoup de types d'individuation. Il y a des individuations du type «sujet» [...], mais il y a aussi des individuations de type événement [comme une œuvre d'art]. De tels modes [d'existence] comportent effectivement des individuations sans sujet ${ }^{\mathbf{3 8}}$.
\end{abstract}

Il ne s'agit donc pas de constituer un «sujet», mais bien de créer de «nouvelles possibilités de $v^{3} e^{39}$ ». La subjectivation comme processus créatif, comme ouverture de possibles, ferait donc face aux attaques multiples consistant à capter les individualités pour en faire des entités sans « résidus» (pleinement «valorisables »). Plutôt que de chercher à partir d'un impossible dehors de la technique des règles de vie, il s'agit ici de penser depuis les formes computationnelles (réseaux, programmes, etc.) pour en dégager de quoi construire collectivement des façons d'habiter notre époque. Ainsi, au contraire des logiciels dominants et uniformisants, les «subjectivités computationnelles» dont parle David M. Berry sont forcément plurielles:

Il s'agit de savoir quels nouveaux modes de connaissances collectives les logiciels peuvent constituer $[\ldots]$ de façon à rendre possible quelque chose $[\ldots]$ comparable à une pensée $[\ldots]$ capable de générer de nouvelles idées, formes de pensée, théories et pratiques ${ }^{40}$.

Cette position part de l'idée stimulante que c'est depuis nos machines qu'il nous faudra trouver comment « déplier la ligne du Dehors» (Deleuze) que constituent les programmes numériques, avant qu'ils ne « dévorent le monde ${ }^{41}$ ». Nous rejoignons ici la pensée de Gilbert Simondon quand il dit qu'«il est nécessaire que l'objet technique soit connu en lui-même pour que la relation de l'homme à la machine devienne stable et valide: d'où la nécessité d'une culture technique ${ }^{\mathbf{4 2}}$ » capable de se représenter le «centre de l'opération ${ }^{\mathbf{4 3}}$ » des machines (ce que nous appelons aujourd'hui «boîte noire»). Pour autant, et c'est en cela que la pensée de Simondon est riche d'enseignements pour notre époque, une machine perfectionnée n'est pas une machine isolée de l'homme, fonctionnant sans lui de façon autonome. C'est même tout le contraire:

Le véritable perfectionnement des machines [...] correspond non pas à un accroissement de l'automatisme, mais au contraire au fait que le fonctionnement d'une machine recèle une certaine marge d'indétermination. [...] La machine qui est douée d'un haut niveau de technicité est une machine ouverte $[\ldots]^{\mathbf{4 4}}$.

Ainsi, ce que dénonce David M. Berry, c'est bien le développement de certains types de machines 
(programmes) avec lesquelles nous ne pouvons strictement rien faire, ou peu faire, ou ne rien faire qui n'ait déjà été anticipé - des machines qui «rendent service» de façon si parfaitement programmée qu'aucune marge de manœuvre ne sera possible, mettant ainsi en défaut toute conduite technique. Contre l'automatisation issue des sciences comportementales, il nous faut donc œuvrer à rechercher et à créer des «marges d'indétermination » au sein de nos rapports aux machines. En ce sens, les pensées autour des «subjectivités computationnelles » peuvent se comprendre comme des actualisations hybrides et collectives de ce que Simondon appelait «individuation ». Comme nous l'avons vu, la délégation de la rationalité humaine à des machines pose des problèmes considérables, et c'est pourquoi David $M$. Berry voit juste lorsqu'il insiste sur l'importance de comprendre et de déconstruire les implications et les conditionnements masqués des programmes. Par là, nous pouvons dire que les «subjectivités computationnelles» ne se constituent comme subjectivités qu'en raison de la compréhension critique de leur milieu technique:

Il est [...] essentiel que la constellation de concepts qui sous-tend et soutient le capitalisme computationnel soit rigoureusement contestée et que les logiciels qui le rendent effectif soient hackés, détricotés et défaits ${ }^{45}$

\section{Pour des consciences « appareillées »}

Pour autant, et cela n'est pas contradictoire, si les machines ne se perfectionnent qu'en tant qu'elles recèlent des «marges d'indétermination » (Simondon) laissant place à l'action humaine, il nous importe donc aussi qu'elles ne fassent pas systématiquement ce qu'on leur assigne. Chez Foucault, comme le dit Deleuze, la «subjectivation [...] est éthique et esthétique, par opposition à la morale qui participe du savoir et du pouvoir ${ }^{46} »$. Ainsi, il n'y aurait de subjectivation possible dans le numérique que pour autant que nous serions prêts à mettre en suspens ce qui nous constitue comme sujets stables assumant une position de maîtrise (raisonnables, doués de savoirs et de pouvoir, etc.). Le paradoxe consiste alors à «faire communauté » en mettant de côté, fût-ce provisoirement, le processus d'«assujettissement social». Le sujet proprement assujetti, que ce soit par des institutions ou par des machines, est supposé «responsable »: il est reconnu comme sujet dans la mesure où il « répond » de façon adéquate aux stimuli - c'est-à-dire dans la mesure où il compute selon les modes de computations déjà admis, déjà programmé. Or, ce dont ont besoin des subjectivités computationnelles, c'est de pouvoir computer différemment de ce qu'on attend d'un sujet - c'est en quelque sorte de pouvoir computer sans sujet! Mais comment une computation «sans sujet » pourrait-elle ne pas être «déshumanisée ${ }^{47} »$ ?

C'est ici qu'entre en jeu la dimension esthétique de la subjectivation, essentielle à l'être humain. Comme le dit Pierre-Damien Huyghe: «si nous tenons bien évidemment au langage et à la capacité de signifier, nous tenons aussi à la puissance brute de la sensibilité ${ }^{\mathbf{4 8}}$ ». L'expérience esthétique a ceci de singulier qu'elle suspend la subjectivité, à savoir la maîtrise et la capacité 
d'un sujet d'être extérieur à un objet. Dans l'analyse qu'en donne Pierre-Damien Huyghe, les appareils apparus à partir de la fin du XIX $X^{\mathrm{e}}$ siècle (l'appareil photographique, la caméra) sont ainsi spécifiques de par leur faculté à enregistrer le visible sans qu'un sujet ne puisse jamais tout à fait les contrôler:

Ce qu'il faut comprendre, c'est qu'il y a et qu'il n'y a pas sujet. [...] Une fois que l'appareil est mis en œuvre, une fois qu'il est ouvert, une fois qu'il tourne, ce sujet s'absente : il est exclu par la mécanique même de l'opération. C'est précisément de la résistance à cette absence et à cette exclusion qu'est faite l'histoire de la photographie et du cinéma ${ }^{49}$

Comme chacun d'entre nous en aura déjà fait l'expérience, les appareils n'enregistrent jamais tout à fait ce que nous avions prévu dans nos efforts de maîtrise programmatrice. C'est ce décalage entre une intention et un «temps » où une machine travaille seule qui fait la spécificité d'un appareil, en tant qu'il suspend la faculté d'un sujet souverain à assigner l'expérience dans du langage. Le terme qui convient à cette expérience ne serait pas celui de subjectivité, mais de «conscience»:

Mais quelle conscience? En fait, celle qui se trouve, se fait ou se prend - et ne peut que se trouver, se faire ou se prendre - à l'écart du sujet. [...] Quand cette parution [de la conscience] se produit, $[\ldots]$, c'est que quelque chose qui n'était pas dans mon point de vue entre dans mon champ et se fait de la place. [...] Pour reprendre un mot que je viens d'utiliser, [la conscience] suppose bien [...] la marque [...] d'un «écart», écart entre la capacité de compréhension où je me trouve comme sujet et l'expérience qui arrive par ailleurs à l'être sensible que je suis plus globalement ${ }^{\mathbf{5 0}}$.

Les «appareils» sont donc pris dans une tension entre des expériences sensibles qui suspendent toute subjectivité et une volonté de les assujettir (de les constituer en objets contrôlables). Le passage des appareils d'enregistrement et de reproduction aux machines de computation aura peut-être consisté à aller dans le sens d'une maîtrise du sujet sur la technique, contrôle tellement exacerbé que cette technique est désormais détentrice du sens, du logos (techno-logie). C'est pourquoi la «culture du code » est importante, en tant que la compréhension des agencements symboliques peut nous aider à les subvertir afin de développer d'autres langages, d'autres formes de vies, d'autres façons de faire communauté.

Comme on le sait au moins depuis Gödel, les programmes numériques ne pourront jamais être totalement infaillibles en raison de l'impossibilité de simuler tous les paramètres d'exécution (ils finiront toujours par «planter»). Sur un autre plan, des auteurs comme Jacques Derrida nous ont appris que toute écriture est impure, qu'elle ne se redouble pas sans «supplément ${ }^{\mathbf{5 1}}$ », sans reste, sans ambiguïté. C'est pourquoi il nous semble essentiel de ne pas limiter la computation à une logique formelle mathématique qui serait transparente à elle-même, promesse de rapidité et d'efficacité sans bornes. Alors que les ordinateurs se sont historiquement inventés dans le prolongement de modèles cognitifs comportementaux, il nous faut œuvrer à en faire des appareils, c'est-à-dire des machines qui soient ouvertes à de multiples formes de lecture, d'écriture et d'expériences esthétiques. Une telle visée implique un travail de réception critique 


\begin{abstract}
des logiciels rendant effectif le «capitalisme computationnel» (David M. Berry), mais elle nécessite également des démarches de design proposant de nouvelles méthodes d'approches «se positionn[ant] à la fois contre l'idée de l'outil transparent et du génie romantique du créateur, mais aussi contre la machine comme entité autonome ${ }^{52}$. » À rebours de l'injonction contemporaine à mettre du signifiant partout ${ }^{53}$, plaidons pour que nos existences appareillées puissent aussi développer des poches de vide.
\end{abstract}

\title{
Notes
}

1 Dominique Cardon, Antonio A. Casilli, Qu'est-ce que le digital labor?, INA, coll. Études et controverses, Paris, 2015.

2 Voir: Yves Citton (dir.), L'économie de l'attention, La Découverte, coll. Sciences humaines, Paris, 2014.

3 Voir: Anthony Masure, «Le passage du logiciel à l'application», dans: Le design des programmes, des façons de faire du numérique, thèse dirigée par Pierre-Damien Huyghe, Université Paris 1 Panthéon-Sorbonne, 2014, [En ligne], anthonymasure.com/softphd.com/these/web2/logiciel-application

4 Les publicités pour l'iPad où l'on voit des corps allongés voués à la consommation passive peuvent être vues comme le symbole du passage historique de l'informatique comme accès à la connaissance à l'informatique comme divertissement généralisé.

5 Comme en témoigne le succès économique du design «de service».

6 Jean-Michel Salanskis, Le monde du computationnel, Les Belles Lettres, coll. Encre Marine, Paris, 2011, p. 154: «C'est ce que signifie le concept de programme, exactement: un devenir qui obéit à un schéma idéal lui-même agencé selon des règles.»

7 David M. Berry, «Subjectivités computationnelles», Multitudes, n59, 2015, p. 196-205.

8 Ibid., p. 200-201.

9 Gottfried Wilhelm Leibniz, La Monadologie [1714], éd. établie par Émile Boutroux [1881], UCAQ, coll. Les classiques des sciences sociales, Chicoutimi, 2002, §7.

10 lbid., $§ 51$.

11 ibid., $§ 56-60$.

12 «To make the world a better place», comme illustré dans la série parodique Silicon Valley créée par Mike Judge en 2014.

13 Éric Sadin, La société de l'anticipation, Inculte, coll. Essais, Paris, 2011. Voir également, du même auteur: Softlove, Galaade, Paris, 2015.

14 Hannah Arendt, Condition de l'homme moderne [1958], trad. de l'anglais par Georges Fradier, Pocket, coll. Évolution, Paris, 2001, p. 335.

15 ibid., p. 336.

16 Ibid., p. 337.

17 lbid., p. 356.

18 Voir le commentaire que propose Franck Varenne de l'article de von Neumann «Théorie générale et logique des automates» [1948] dans: Qu'est-ce que l'informatique?, Vrin, Paris, 2004, p. 73: «ce qui rend les calculateurs numériques programmables désormais nécessaires [...], c'est cette supériorité qu'ils ont, par rapport aux calculateurs analogiques, de réduire considérablement le rapport "bruit sur signal".»

19 André Leroi-Gourhan, «Le geste et le programme» [1965], dans: Le geste et la parole, tome 2, La mémoire et les rythmes, Albin Michel, coll. Sciences d'aujourd'hui, 1988.

20 Voir par exemple: Gunther Anders, La Menace nucléaire. Considérations radicales sur l'âge atomique [1958-1986], Paris, Le 
serpent à plumes, 2006.

21 Judy L. Klein, Rebecca Lemov, Michael D. Gordin, Lorraine Daston, Paul Erickson, Thomas Sturm, Quand la raison faillit perdre l'esprit. La rationalité mise à l'épreuve de la Guerre froide [2013], trad. de l'anglais par Jean-François Caro, Zones Sensibles, Bruxelles, 2015, p. 230.

22 Herbert A. Simon, «A Behavioral Model of Rational Choice» [1955], ibid., p. 98: «Cet essai explorait plusieurs méthodes de modélisation du processus du choix rationnel qui prenaient en compte les limites computationnelles.»

23 Ibid., p. 230.

24 Voir notamment les pages consacrées à Robert F. Bales dans: «La «situation » dans les sciences comportementales durant la Guerre froide », ibid., p. 149-158.

25 Paul N. Edwards, Un monde clos. L'ordinateur, la bombe et le discours politique de la Guerre froide [1996], B2, coll. Territoires, Paris.

26 Herbert A. Simon, «Notes - on Rationality» [1965], cité dans: Quand la raison faillit perdre l'esprit, op. cit., p. 149: «cette formule [de Simon] saisit à merveille l'essence de notre sujet [et] résume à la perfection le rôle de la situation: maintenir la mélasse dans son récipient, afin que l’on puisse étudier et déterminer les composants spécifiques de chaque élément. »

27 David M. Berry, «Subjectivités computationnelles», op. cit.

28 Ivan Illich, La convivialité [1973], Seuil, coll. Points Essais, Paris, 2003.

29 Gilles Deleuze, «Post-scriptum sur les sociétés de contrôle», dans: Pourparlers, Minuit, Paris, 1990, p. 247: «la nouvelle médecine [...] qui dégage des malades potentiels et des sujets à risque [...] substitue au corps individuel ou numérique le chiffre d'une matière «dividuelle» à contrôler.»

30 Félix Guattari, «De la production de subjectivité », dans: Chaosmose, Paris, Galilée, coll. La philosophie en effet, Paris, 1992, p. 11-52.

31 Yves Citton (à propos de Maurizio Lazzarato) dans: Gestes d'humanités. Anthropologie sauvage de nos expériences esthétiques, Paris, Armand Colin, coll. Le temps des idées, Paris, 2012, p. 70. Voir aussi : Gilles Deleuze et Félix Guattari, Mille plateaux, Paris, Minuit, 1980, p. 570 et 572.

32 Maurizio Lazzarato, «La machine», introduction au livre Tausend Maschinen de Gerald Raunig, EIPCP, [En ligne], eipcp.net/transversal/1106/lazzarato/fr

33 Maurizio Lazzarato, La fabrique de l'homme endetté, éd. Amsterdam, Paris, 2011, p. 111.

34 Cette opération de pointage recoupe ce que Hannah Arendt disait à propos du « réseau mathématique » qui «ne dépend pas de qualités et ne requiert autre chose qu'une multitude d'objets donnés ». Voir: Condition de l'homme moderne, op. cit., p. 337.

35 Giorgio Agamben, Qu'est-ce qu'un dispositif? [2006], trad. de l'italien par Martin Rueff, Payot \& Rivages, coll. Petite Bibliothèque, Paris, 2007, p. 43-44: "Ce qui définit les dispositifs auxquels nous avons à faire dans la phase actuelle du capitalisme est qu'ils n'agissent plus par la production d’un sujet, mais bien par des processus [...] de désubjectivation. »

36 Maurizio Lazzarato, «La machine», op. cit. : «Dans l'asservissement machinique, [...] nous fonctionnons comme des composantes du dispositif, comme des éléments d'input / output, comme des simple relais [...], qui font passer et/ou empêchent le passage [...] des signes.»

37 Evgeny Morozov, «La prise de pouvoir des données et la mort de la politique», Blog de Paul jorion, août 2014, [En ligne], anthonymasure.com/pauljorion.com/blog/2014/08/25/la-prise-de-pouvoir-par-les-donnees-et-la-mort-de-la-politiqueparevgeny-morozov

38 Gilles Deleuze, entretien avec Claire Parnet, «Portrait de Foucault» [1986], dans: Pourparlers, op. cit., p. 158.

39 Ibid., p. 160.

40 David M. Berry, op. cit.

41 Marc Andreessen, «Why Software Is Eating The World», Wall Street Journal, août 2011, [En ligne], anthonymasure.com/wsj.com/articles/SB10001424053111903480904576512250915629460

42 Gilbert Simondon, Du mode d'existence des objets techniques [1958], Aubier, Paris, 2012, p. 82. 
43 Ibid., p. 243-244.

44 Ibid., p. 11

45 David M. Berry, op. cit.

46 Ibid., p. 155.

47 David M. Berry, op. cit., : «Cette pensée computationnellement assistée n'a nul besoin d'être déshumanisante. »

48 Pierre-Damien Huyghe, Le cinéma Avant Après, Saint-Vincent-de-Mercuze, De l'Incidence, 2012, p. 25.

49 Ibid., p. 14-15.

50 Ibid., p. 17

51 Voir notamment: Jacques Derrida, De la Grammatologie [1967], Paris, Minuit, coll. Critique, 1974.

52 Alexandre Leray, Stéphanie Vilayphiou (STDIN), «Écrire le design. Vers une culture du code», B42, Back Cover, n 4, Paris, 2011, p. 43. Cette citation fait référence au travail du studio de design graphique Lakfon qui «assume et utilise la matérialité du code [...] mais également celle [du] «paracode» (noms de fichiers, arborescences, commentaires, journaux d'erreurs...). Cette approche rompt radicalement avec l'idée d'un environnement de design unifié comme le proposent les suites logicielles.»

53 Jean-Michel Salanskis, Le monde du computationnel, op. cit., p. 191-192: «Le passage de nos modes existentiels ou de nos modes sociaux au computationnel ne fait jamais autre chose que mettre en relief une inhérence de la chose combinatoire, arithmétique, syntaxique, linguistique, symbolique à ces modes.» 